\title{
Diversité floristique et infiltration humaine de la forêt classée de la Besso (Côte d'Ivoire)
}

\author{
Justin KASSI N'Dja', TUO Yetchawa' ${ }^{1}$ \& ZO-BI Irie Casimir² \\ 1 : Laboratoire de Botanique, UFR Biosciences, Université Félix Houphouët-Boigny, 22 BP 582 Abidjan 22, Université \\ FHB (Biosciences), Côte d'Ivoire \\ 2: INP-HB de Yamoussoukro (Côte d'Ivoire), BP. 2661 Yamoussoukro \\ E-mail de l'auteur correspondant : kassindja@yahoo.fr
}

Original submitted in on $13^{\text {th }}$ March 2017. Published online at www.m.elewa.org on $30^{\text {th }}$ June 2017 https://dx.doi.org/10.4314/jab.v114i1.3

\begin{abstract}
Objectif : Contribuer à une meilleure connaissance de la flore de la forêt classée de la Besso et de caractériser les populations infiltrées et les cultures pratiquées dans cette forêt classée.

Méthodologie et résultats : La forêt classée de Besso (Côte d'Ivoire) couvre 23100 ha sur est une forêt dense semi-décidue à Celtis spp. et Triplochiton scleroxylon. La création de clairières culturales entre 1966 et 2012 malgré le classement de la forêt est à l'origine d'une mosaïque de végétations secondaires incluses dans une matrice de forêt « primaire » que nous avons plus particulièrement étudié. Les relevés de surfaces couplés aux inventaires itinérants ont permis d'inventorier 474 espèces, qui se repartissent en 330 genres et 91 familles. Les familles les plus abondantes sont Fabaceae, les Rubiaceae, les Euphorbiaceae, les Moraceae, les Malvaceae, les Meliaceae, les Moraceae, les Annonaceae, les Sapindaceae et les Poaceae. Parmi ces espèces, $79 \%$ sont des phanérophytes et $3 \%$ des hémicryptophytes.

Conclusion : Malheureusement, cette forêt est en progressive dégradation à cause des activités humaines qui menacent la biodiversité végétale et animale de cette forêt. Ainsi, pour réduire le phénomène de dégradation des forêts en générale, plusieurs actions de gestion durable pourraient être entreprises par l'État, les ONG et la population. Notamment par la création des zones de conservation de plantes par des mises en défens de jachères.
\end{abstract}

Mots clés : Biodiversité végétale, Forêt classée de la Besso, Côte d'Ivoire.

Floristic diversity and human impact on Besso forest in Ivory Coast

Objective: To contribute significantly to the knowledge of the flora of Besso forest and to evaluate the human impact.

Methodology and results: The Besso forest in Ivory Coast) covers 23100 ha and determines a potential vegetation corresponding to the Celtis spp.-Triplochiton scleroxylon semi-deciduous tropical forest. Shifting cultivation between 1966 and 2012 has led to a mosaic of secondary plant communities that are included in an old-growth forest matrix. For hundred seventy for (474) species were recorded in this forest during the field investigations, distributed among 330 genera and 91 families. The most common families were Fabaceae, Rubiaceae, Euphorbiaceae, Moraceae, Malvaceae, Meliaceae, Moraceae, Annonaceae, Sapindaceae and Poaceae. Among those species, $79 \%$ and $3 \%$ are phanerophytes and hemicryptophytes, respectively. 

(Côte d'Ivoire)

Conclusion : Unfortunately, this forest is gradual degradation due to uncontrolled human's activity. Thus, to reduce the degradation phenomenon of the forets in general, several actions of sustainable managements could be undertaken by the state, Non-Governmental Organisation (NGO) and the population. In particular by the creation of preservation zones of plants, the creation of collective gardens of plants.

Key words: Plant biodiversity, Besso forest, Ivory Coast.

\section{INTRODUCTION}

L'impact des activités humaines sur la végétation forestière actuelle commence à être bien documenté pour les régions tempérées, il n'en est pas de même pour les forêts tropicales, pour lesquelles les approches historiques et écologiques restent très souvent déconnectées (Decocq, 2003). L'irréversibilité des altérations anthropogènes supposée pour les forêts tempérées (Dupouey et al., 2002) mérite d'être examinée dans le cas des forêts tropicales, dans la mesure où leur extraordinaire capacité de résilience (Senterre, 2005), vient changer la donne. II est en effet essentiel de comprendre tous les déterminants de la diversité végétale actuelle pour ces écosystèmes, qui figurent parmi les plus menacés de la planète. Ainsi, pour Puig (2001), les forêts tropicales humides sont les écosystèmes forestiers les plus complexes et les plus diversifiés de la planète, au point qu'un nombre important d'entre-elles fait partie des «points chauds » de la biodiversité mondiale et constitue une priorité en matière de conservation de la nature (Myers et al., 2000). En Côte d'Ivoire, selon Aké-Assi (1984; 1988), la flore ivoirienne est bien connue et est estimée à 3882 espèces. Soixante sept (67) de ces espèces signalées dans cette flore n'ont, jusqu'à ce jour, été observées hors des limites de la Côte d'lvoire (Aké-Assi, 1988). Ce qui en fait l'un des «points chauds» de la biodiversité («biodiversity hotspot ») à l'échelle planétaire. Cependant, selon Balaguru et al. (2006), malgré la mise en place de diverses mesures de protection de la faune et de la flore à travers les Parcs Nationaux, les Réserves biologiques, les Forêts classées, la déforestation

\section{MATERIEL ET METHODES}

Zone d'étude : La forêt privée ou domaine soumis à notre étude (464,02 hectares), est située dans la Région administrative de la Mé au Sud-est de la Côte d'lvoire $\left(6^{\circ} 10^{\prime}\right.$ et $6^{\circ} 30^{\prime}$ Nord, $3^{\circ} 35^{\prime}$ et $3^{\circ} 50^{\prime}$ Ouest). La forêt étudiée est une forêt dense humide semi-décidue. Elle s'est accrue et continue de croître aussi bien à l'intérieur qu'à l'extérieur des aires protégées, au profit de l'agriculture avec pour corolaire la dégradation et la disparition de nombreuses espèces animales et végétales. La forêt classée de la Besso, fruit de la convention de partenariat ( $n^{\circ} 001-\mathrm{A} 3-2004$ du 8 septembre 2004) entre la SODEFOR et la société de transformation du bois INPROBOIS bénéficie d'un plan d'aménagement. Cependant, malgré les efforts consentis par la SODEFOR et la société INPROBOIS pour une gestion durable du massif forestier de la Bésso, fort est de constater que les infiltrations clandestines dans cette forêt, continuent et menacent l'existence même de cette forêt au profit des plantations agricoles (INPROBOIS, 2013). La biodiversité de cette forêt est menacée par le braconnage, la démographie, la perte d'habitats (les défrichements agricoles)... La survie de cette forêt est préoccupante. Comment décrire de façon systématique la biodiversité végétale de cette forêt encore mal connue et très partiellement quantifiée ? Le préalable indispensable est l'inventaire des espèces végétales qui composent cette biocénose. Ainsi, l'objectif général de cette étude est donc de contribuer de manière significative à une meilleure connaissance de la flore de la forêt classée de la Bésso et d'évaluer partiellement l'impact des activités humaines sur cette flore. Plus spécifiquement, nous allons tenter de répondre aux questions suivantes : 1) - quelle est la flore de cette forêt? 2) - quelle est l'impact des activités humaines sur cette flore?

est caractérisée, par Celtis spp. et Triplochiton scleroxylon K. Schum. (Guillaumet \& Adjanohoun, 1971) et correspond à l'association à Celtis-Triplochiton. La variante à Nesogordonia papaverifera (A. Chev.) R. Capuron et Khaya ivorensi A. Chev., qui est une forme de 
transition avec la forêt sempervirente (Guillaumet \& Adjanohoun, 1971). Mangenot (1955) caractérise les forêts semi-caducifoliées par la présence exclusive d'espèces du genre Celtis (Celtidetalia). Selon White (1986), il s'agit d'une forêt dense mésophile.

Méthode: Le plan d'échantillonnage a été conçu de manière à placer des relevés de surface dans tous les types de végétation rencontrés sur le site à savoir les forêts galeries $(n=6)$, les forêts primaires sur sol ferralitiques $(n=15)$, le, les forêts primaires sur sol hydromorphes $(n=14)$ et les jachères $(n=25)$. La taille des 60 relevés était de $50 \mathrm{~m} \times 30 \mathrm{~m}\left(1500 \mathrm{~m}^{2}\right)$. Une telle surface permettra de prendre en compte les aires minimales des communautés végétales en forêts tropicales (Senterre, 2005). Le relevé de végétation pour cette étude a consisté à recenser toutes les espèces vasculaires présentes dans la surface-échantillon. Des inventaires itinérants complémentaires ont été menés dans toutes les parties de la forêt y compris les cultures. Ces inventaires ont permis de compléter la liste floristique. Toutes les espèces ont été identifiées. La nomenclature adoptée est celle de APG III (2009). Les noms des espèces ont été actualisés grâce aux travaux de Lebrun \& Stork (1991-1997). L'objectif de l'étude était aussi de caractériser les populations infiltrées (les Chefs

\section{RESULTATS}

Richesse et composition floristique : La flore de la forêt classée de Besso compte 474 espèces, réparties en 330 genres et 91 familles. Les familles les plus abondantes en espèces, sont les Fabaceae (exPapilionaceae, ex-Caesalpiniaceae et ex-Mimosaceae). Elles renferment 47 espèces (10\%). Elles sont suivies
d'Exploitations agricoles), les cultures pratiquées dans la forêt classée de la Besso et de connaitre l'évolution du nombre des Chefs d'Exploitations en forêt classée de la Besso. La collecte des données a été faite par la société INPROBOIS. Les enquêtes ont constitué au remplissage d'un questionnaire créé à cet effet et au levé des parcelles agricoles. La technique d'enquête utilisée est l'interview individuelle. Les enquêtes ont été réalisées par deux équipes de trois (03) personnes comprenant, un élève-ingénieur des techniques, forestières et deux interprètes.

Analyse des données : Le nombre d'espèces, de genres et de familles de toutes les espèces végétales rencontrées lors des inventaires dans le site d'étude ont été déterminé. Par la suite, les types biologiques ont été complétés en nous référant à Raunkiaer (1934). Les types chorologiques (Whites, 1986) et les modes de disséminations des espèces ont été précisés. Ces paramètres ont permis d'avoir une idée globale de la richesse, de la composition floristique et de la structure de la végétation. Par confrontation de notre liste à celles de Aké-Assi (1984) et de l'UICN (2009), nous avons mis en évidence le statut particulier de certaines espèces récences. Les espèces à valeur commerciale ont été recensées.

des Rubiaceae avec 30 espèces (7\%) puis les Apocynaceae (espèces 27), les Euphorbiaceae (27 espèces), les Malvaceae (23 espèces), les Meliaceae (16 espèces), les Moraceae (13 espèces), les Annonaceae (espèces 12), les Poaceae (11 espèces) et les Sapindaceae (11 espèces), (Figure 1).

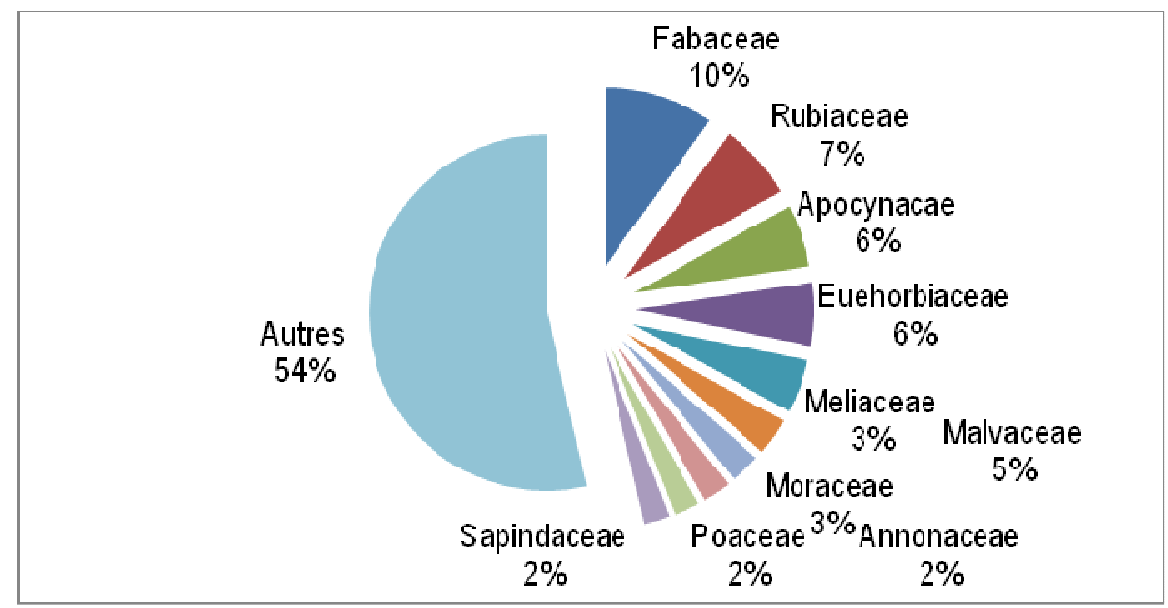

Figure 1 : Spectre de répartition des familles botaniques de la forêt classée de la Besso 
Types biologiques: Les espèces recensées dans la forêt classée de la Besso appartiennent à 8 grands types biologiques et de quelques autres types biologiques très peu représentés (Figure 2). Le spectre biologique de la forêt classée de la Besso, se caractérise par la dominance des phanérophytes. Mais pris séparément, nous constatons l'abondance des mésophanérophytes $(47 \%)$ et les mégaphanérophytes $(19 \%)$. Les nanophanérophytes sont relativement peu représentés (9\%). Tous les types biologiques peuvent être regroupés en 4 types morphologiques (espèces arbustives: 186 espèces soit $39,32 \%$; espèces lianescentes: 149 espèces soit $31,50 \%$; espèces arborées : 124 espèces soit $26,21 \%$; espèces herbacées : 75 espèces soit 15,85 $\%)$.

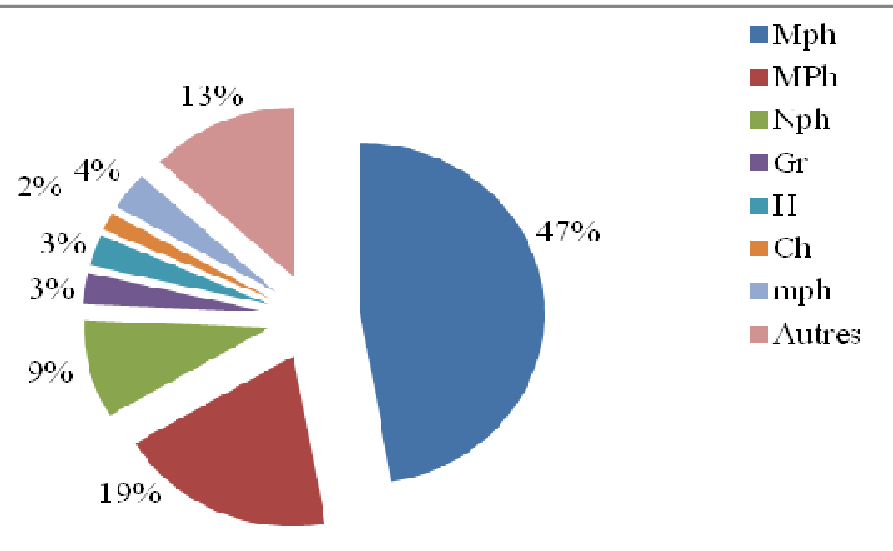

Figure 2 : Spectre des types biologiques de la flore de la Besso

Légende : mph : microphanérophytes, Mph : mésophanérophytes, Nph : nanophanérophytes, MPh :

Mégaphonérophytes, $\mathrm{Gr}$ : géophytes, $\mathrm{H}$ : hémicryptophytes, $\mathrm{Ch}$ : chaméphytes.

Affinités chorologiques : La flore de la forêt classée de la Besso est constituée majoritairement d'espèces guinéo-congolaises (GC) soit 320 espèces $(67,51 \%)$. Les espèces afro-tropicales (AT), suivent avec 40 espèces (soit $8,44 \%$ de l'effectif total). Les espèces endémiques Ouest-africaines sont au nombre de 35 espèces (soit 7,38 $\%$ ). Les espèces de transition forêt-savanes ou de liaison guinéo-congolaises-soudano-zambéziennes (GC-SZ), sont quant à elles au nombre de 32 espèces (soit 6,75 $\%)$. La répartition des autres espèces inventoriées selon la classification de White se présente comme suite: pantropicales (Pan, 23 espèces, soit 4,85\%) ; espèces plurirégionales africaines (AT et $P A, 15$ espèces, soit 3,16 $\%$ ), les afro-américaines (AAM, 02 espèces, soit $0,42 \%$ ). Les espèces endémiques ivoiriennes ( $\mathrm{GCi}: 2$ espèces, soit $0,42 \%$ ) sont au nombre de deux (Figure 3).

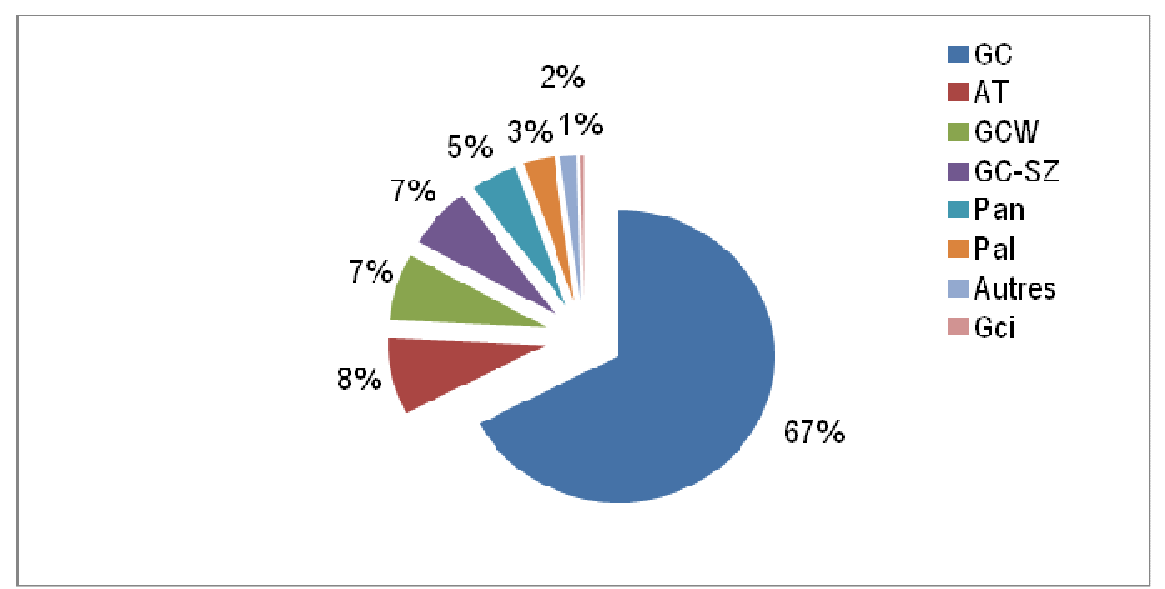

Figure 3 : Spectre des types phyrogéographiques de la flore de la forêt classée de la Besso Légende : GC : Guinéo-congolaises, GC-SZ : Transition GC-SZ, AT : afro-tropicales ; Pan : Pantropicales ; Pal : plurirégionales africaines, soudano-zambéziennes : $S Z$ 
Mode de dissémination : L'endozoochorie avec 276 espèces soit $66 \%$ de l'effectif total est le mode de dissémination le plus représentatif des espèces du site (Figure 4). Elle est suivie de l'anémochorie avec 83 espèces soit $20 \%$ et de l'épizoochorie (42 espèces, soit $10 \%$ ). Les espèces barochores et Hydrochores (avec 8 espèces chacun, soit $4 \%$ de l'effectif total) sont moins représentées dans le site.

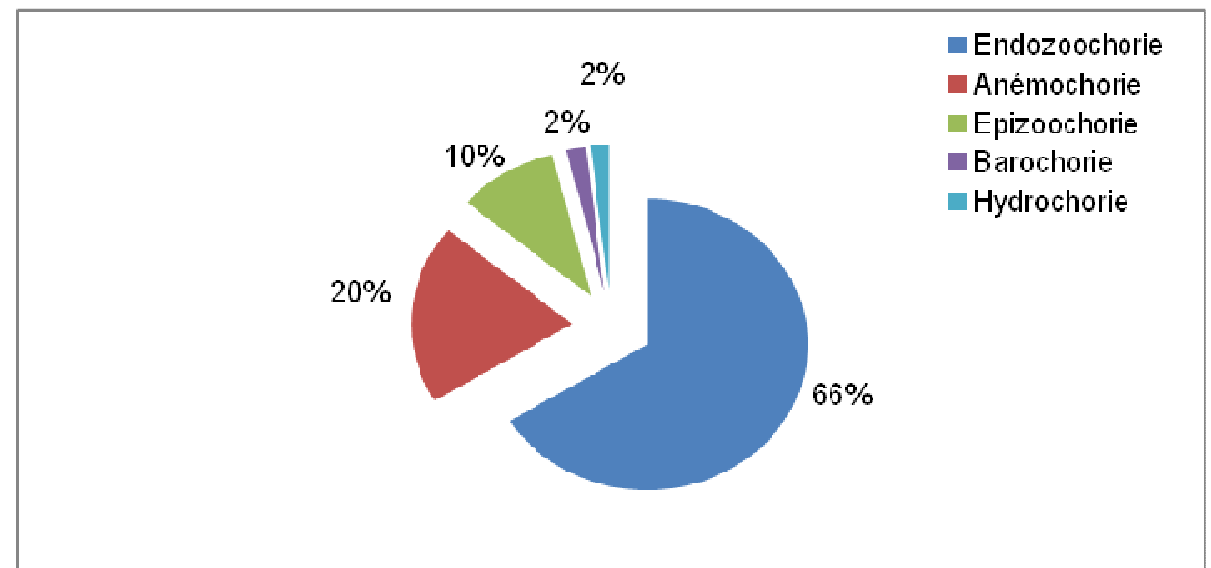

Figure 4 : Spectre de dissémination des diaspores de la forêt classée de la Besso

Valeur de conservation de la biodiversité du domaine Espèces à statut particulier: Parmi les espèces recensées dans la forêt classée de la Besso, 35 (soit 7,38 $\%$ de l'effectif total) sont endémiques Ouest africaines (GCW). Deux autres espèces sont endémiques ivoiriennes (GCi). Parmi ces espèces, 26 figurent sur la liste rouge de l'UICN, dont 21 espèces sont vulnérables (VU) et 1 espèce à risque bas (L.R). La forêt classée renferme également 1 espèce considérée comme plante rare, devenue rare ou ne se rencontrant qu'exceptionnellement (Tableau 1).

Tableau 1 : Liste des espèces à statut particulier de la forêt classée de la Besso

\begin{tabular}{l|l|l|l|l|l}
\hline \multicolumn{5}{l}{} & \multicolumn{2}{l}{ Statut } \\
\hline $\mathbf{N}^{\circ}$ & Nom scientifique & Nom vernaculaire & Famille & UICN & AKE Assi \\
\hline 1 & Afzelia africana & Lingué & Fabaceae & VU & \\
\hline 2 & Albizia ferruginea & latandza & Fabaceae & VU & \\
\hline 3 & Anopyxis klaineana & Bodioa & Rhizophoraceae & VU & \\
\hline 4 & Antrocaryon micraster & Akoua & Anacardiaceae & VU & \\
\hline 5 & Copaïfera selikounda & Etimoé & Fabaceae & EN & \\
\hline 6 & Entandrophragma angolense & Tiama & Meliaceae & VU & \\
\hline 7 & Entandrophragma canllei & Kosipo & Meliaceae & VU & \\
\hline 8 & Entandrophragma cylindricum & Aboudikro (Sapelli) & Meliaceae & VU & \\
\hline 9 & Eribroma oblonga & Bi & Sterculiaceae & VU & \\
\hline 10 & Garcinia kola & Aouolié & Salicaceae & LC & \\
\hline 11 & Guarea cedrata & Bossé & Meliaceae & VU & \\
\hline 12 & Guirbourtia ehie & Amazakoué & Fabaceae & VU & \\
\hline 13 & Irvingia gabonensis & Boborou & Irvingiaceae & IE & \\
\hline 14 & Khaya anthotheca & Acajou blanc & Meliaceae & VU & \\
\hline 15 & Khaya grandifolia & Acajou à grandes feuilles & Meliaceae & VU & \\
\hline 16 & Khaya ivorensis & Acajou & Meliaceae & VU & \\
\hline & & & & & \\
\hline
\end{tabular}


N'Dja et al., J. Appl. Biosci. 2017 Diversité floristique et infiltration humaine de la forêt classée de la Besso (Côte d'Ivoire)

\begin{tabular}{l|l|l|l|l|l}
\hline 17 & Lovoa trichiliodes & Dibétou & Meliaceae & VU & \\
\hline 18 & Milicia excelsa & Iroko & Moraceae & VU & AA \\
\hline 19 & Nauclea dirrichii & Badi & Rubiacées & VU & \\
\hline 20 & Nesogordonia papaverifera & Kotibé & Sterculiaceae & VU & \\
\hline 21 & Pterygota bequaertii & Akodiakédé & Sterculiaceae & VU & \\
\hline 22 & Pterygota macrocarpa & Koto & Sterculiaceae & VU & \\
\hline 23 & bombax brevicuspe & Kondroti & Malvaceae & VU & \\
\hline 24 & Teminalia ivorensis & Framiré & Combretaceae & VU & \\
\hline 25 & Thieghemella heckelii & Makoré & Fabaceae & EN & \\
\hline 26 & Triplochiton scleroxylon & Samba & Sterculiaceae & LR & \\
\hline
\end{tabular}

Légende : espèces reconnues comme vulnérables (VU), espèces en danger (EN), espèces qui courent des risques mineurs "LR" de la liste Rouge de UICN, 2009) ; $A A$ = plantes rares, devenues rares et en voie d'extinction ou ne se rencontrant qu'exceptionnellement Aké-Assi (1998).

Espèces ligneuses à valeur commerciale : L'inventaire floristique de la forêt classée de Besso a permis de recenser au total 74 espèces végétales commerciales utilisées comme matières premières dans l'industrie du bois soit $15,65 \%$ des activités espèces inventoriés. Ces espèces sont réparties entre 64 genres regroupés au sein de 24 familles. Les familles les plus représentatives en nombre d'espèces sont celles des Myristicaceae (19 espèces recensées, 25,67 \%), des Méliacées (10 espèces, $14 \%$ ) et des Ochnaceae (8 espèces, $11 \%$ ).

Produits forestiers non ligneuses à valeur commerciale : L'inventaire floristique de la forêt classée de Besso a permis d'identifier au total 18 espèces végétales qui fournissent des produits forestiers non ligneux à valeur commerciales pour les populations riveraines à la forêt. Ces produits sont pour la plupart utilisées dans la médecine traditionnelle soit 3,80\% des espèces inventoriés. Ces espèces sont réparties au sein de 14 familles. Les familles les plus représentatives en nombre d'espèces sont celles des Fabaceae (11 espèces recensées, $15 \%$ ), des Méliacées (10 espèces, 14\%) et des Sterculiaceae (8 espèces, $11 \%$ ).
Dynamique d'infiltration des populations en forêt classée de Besso: La dynamique de l'infiltration paysanne (Figure 5), suivie de 1966 à 2012 par la société INPROBOIS, montre trois principaux pics et des phases de faible infiltration. Ainsi, l'année 1980 correspond au boom du cacao et du café en Côte d'lvoire, ce qui a entraîné une forte pression sur la forêt classée qui est située dans une zone de forte production du cacao. Cette date est suivie d'une autre période. II s'agit des années 1997 où l'on observe une forte baisse correspondant à la prise en main de la forêt classée de la Besso par la SODEFOR et à la volonté du gouvernement de faire sortir les occupants des forêts classées. Dans les années 2000 la figure montre une remontée des infiltrations à la suite des troubles en Côte d'Ivoire. Entre 2010 et 2011 qui correspondent à la phase électorale et la crise qui s'en est suivie ont favorisé l'invasion des forêts classées, en raison de l'absence de l'État selon les populations infiltrées. A partir de 2012 l'on observe une baisse des infiltrations avec la reprise normale dans le pays. 


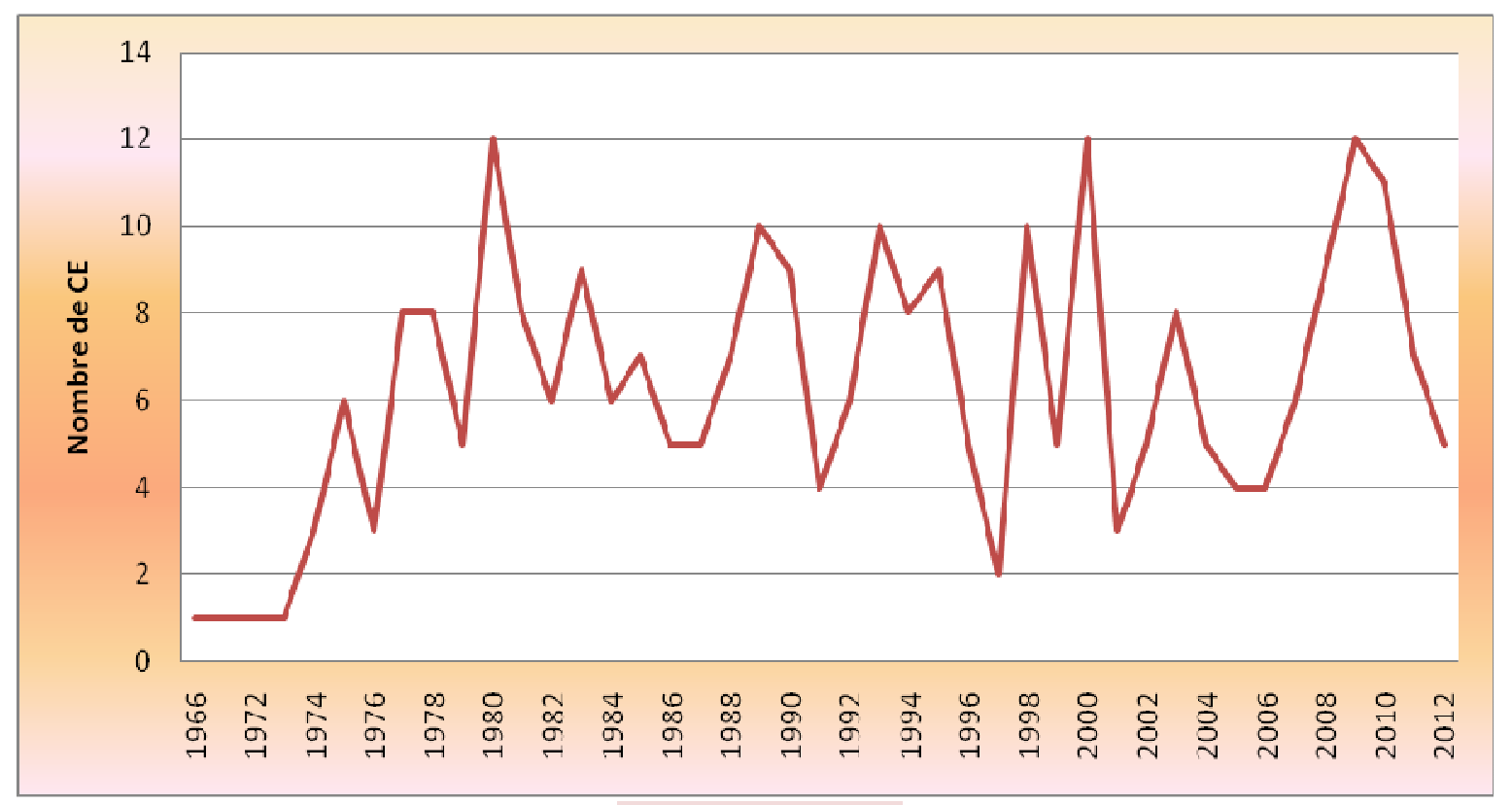

Période (années)

Figure 5 : Évolution du nombre des Chefs Exploitants dans la forêt classée de la Besso de 1966 à 2012 : Légende : CE : Chefs Exploitants

La forêt classée de la Besso compte 587 Chefs d'Exploitants actuellement (CE: Chefs Exploitants: propriétaires des champs). Parmi eux, 469 n'ont pas d'autorisation et 118 possèdent une « cession » familiale. Les superficies occupées par l'agriculture suite aux infiltrations représentent $21 \%$ de la superficie totale de la forêt. Elles sont différentes des chiffres issus du traitement

\section{DISCUSSION}

Richesse et composition floristiques: Nos travaux ont permis d'établir une liste floristique. Les inventaires floristiques menés au sein de la forêt classée ont permis de dénombrer 474 espèces reparties en 330 genres au sein de 91 familles sur une surface de 21565 hectares. Ces espèces constituent une richesse assez importante et représentent $12,21 \%$ de la totalité de la flore ivoirienne telle que recensée par Aké-Assi (1984). Cette flore peut être revue à la hausse car nous n'avons pas eu le temps de parcourir toute la forêt durant ces deux mois de terrain. Néanmoins, nos résultats sont comparables à ceux. Nous avons pu comparer nos résultats à la composition floristique de milieux similaires comme la forêt classée de la Bamo (Agboville) obtenus par Adingra (2013). II ressort, de cette étude floristique, que la flore du site est très diversifiée tant au niveau des espèces, des genres que des familles. Les principaux modes de dispersion des diaspores sont la zoochorie et des images satellitaires. En effet, le traitement de ces images révèle une occupation agricole de 7086 ha dont 5991 ha de cultures pérennes, soit $33 \%$ de la superficie de la forêt. C'est la culture du Cacao qui est la plus pratiquée. Les données sont issues des déclarations des paysans.

l'anémochorie. Les animaux joueraient un rôle assez notable dans la dissémination et indirectement dans le bon fonctionnement du site comme l'a déjà souligné par Adingra (2013), lors de ses travaux dans la forêt classée de la Bamo (Agboville), dans le Sud de la Côte d'Ivoire. Cet auteur, sur une superficie de 255 hectares, avait recensé 414 espèces réparties entre 83 familles et 305 genres. La richesse spécifique de notre site d'étude est moindre par rapport à celui du Parc National de la Marahoué (101 000 ha) situé dans la zone de transition forêt-savane où 607 espèces ont été recensées (N'Da et al., 2008). La richesse de notre site est également inferieure à celle de la forêt classée du Haut-Sassandra 1047 espèces, 538 genres et 114 familles pour une superficie de 102400 ha, selon Kouamé (1998). Les familles botaniques les plus dominantes sur le site sont les Fabaceae, les Rubiaceae, les Euphorbiaceae, les Moraceae, les Malvaceae, les Meliaceae, les Moraceae, 

(Côte d'Ivoire)

les Annonaceae et les Sapindaceae, comme c'est le cas dans la majorité des forêts ivoiriennes. En effet, plusieurs forêts ivoiriennes sont dominées par le même cortège de familles (Bakayoko, 1999). La richesse en Poaceae de la forêt classée de la Besso est probablement dûe à la présence de nombreux champs et jachères postculturales à l'intérieure de la forêt classée. L'importance des espèces zoochores est signalée dans la plupart des travaux portant sur les forêts de Côte d'Ivoire (HoppeDominik, 1999), Le spectre des types de diaspores de la forêt classée révèle la dominance d'espèces endozoochores (66 \%) et anémochores (20\%). Les espèces des forêts de notre site d'étude sont pour la plupart des espèces endozoochores et celles des jachères et champs sont des espèces anémochores. En effet, l'anémochorie constitue une stratégie principale de dissémination pour les plantes des milieux ouverts (Senterre, 2005). Dans les jachères post-culturales, l'abondance d'espèces anémochores revient à l'abondance relative des Fabaceae, des Combretaceae et des Poaceae. La zoochorie est prépondérante et assurée par les oiseaux, les vertébrés frugivores, tels les antilopes, les singes, les rats, les agoutis et les écureuils présents dans la forêt classée de la Besso. Bertault (1986) a en fait les mêmes constats dans ses travaux. Par ailleurs, le mode barochore est peu utilisé par les plantes $(2 \%)$ dans la forêt classée de la Besso. Les résultats similaires ont été trouvés (2,17\%) par Adingra (2013) dans la forêt classée de Bamo. Les espèces à large distribution spatiale sont peu nombreuses. Les espèces afro-tropicales limitées à la zone intertropicale africaine représentent $8 \%$ du spectre. Les espèces guinéennes (guinéo-congolaises GC : $67 \%$; endémiques ouest-africains GCW : $7 \%$; endémiques ivoiriennes GCi : $1 \%)$ sont largement dominantes, avec des proportions qui atteignent $75 \%$.

Valeur pour la conservation de la biodiversité : Signalons que la forêt classée de la Besso compte 27 espèces à statut écologique particulier. Cela montre la nécessité de la protection de cette forêt. L'Azodau (Afzelia bella var. gracilor), de la famille des Fabaceae ex-Cesalpiniaceae, est l'une des espèces endémiques ivoiriennes, observée durant nos travaux dans la forêt calassée de la Besso.

\section{CONCLUSION}

Dans la présente étude, 474 espèces, réparties en 330 genres et 91 familles ont été inventoriées. Les familles les plus nombreuses sont les Fabaceae, les Rubiaceae, les Euphorbiaceae, les Moraceae, les Malvaceae, les Meliaceae, les Moraceae, les Annonaceae, les
Dynamique d'infiltration des Chefs Exploitants en forêt classée de la Besso: L'agriculture, qui est la principale activité de la population riveraine, a été pratiquée en respectant les limites de la forêt, jusqu'au jour où les effets conjugués des immigrations et de l'épuisement des réserves forestières dans le milieu rural environnant, ont amené les riverains à solliciter des autorisations d'occuper et à violer les limites de celles-ci, pour défricher des parcelles dans le périmètre de la forêt classée. Les défrichements clandestins sans autorisation administrative sont qualifiés d'« infiltrations ». II n'existe pas d'implantations agricoles autorisées dans la forêt classée de la Besso (INPROBOIS, 2013). Cependant, nous avons 587 chefs d'exploitants qui exercent dans la forêt. L'occupation du sol a fait ressortir une mosaïque de formations forestières (forêt dégradée et reboisements), de cultures agricoles et de jachères sur l'ensemble de la forêt. Selon INPROBOIS (2013), il est ainsi difficile de localiser des zones de superficie suffisamment importante pour dissocier les différents types de strates pour en faire un traitement spécifique. Ces infiltrations ont eu plusieurs conséquences sur la biodiversité végétale et animale. La chasse est pratiquée mettant en danger les petits mammifères et particulièrement les grands mammifères devenus très rares (grands singes, éléphants...). Selon INPROBOIS (2013) les espèces végétales les plus en danger dans la forêt classée de la Besso sont le Makoré (Thieghemella heckellii: Sapotaceae) et l'Etimoé (Copaïfera selikounda : Fabaceae ex-Caesalpiniaceae). Parmi les espèces vulnérables, certaines ont atteint un seuil critique dans la forêt classée de la Besso. II s'agit d'Afzelia africana Fabaceae ex-Caesalpiniaceae (Lingué), du Lovoa trichiliodes Meliaceae (Dibétou), d'Anopyxis klaineana Rhizophoraceae (Bodioa) et de Pterygota bequaertii Sterculiaceae (l'Akodiaké). Dans un tel contexte, l'on est emmené à s'interroger sur les fondements de la durabilité et la fonctionnalité des Services Ecosystémiques rendus par la forêt classée de la Besso à la population de la Région de la Mé. Si la dynamique d'infiltration paysanne et de prélèvement de la biodiversité n'est pas maîtrisée, elle pourrait impacter négativement les Services Ecosystémiques rendus par la forêt classée de la Besso à la population.

Sapindaceae et les Poaceae. La diversité de la flore de la forêt classée est aussi reflétée par la présence de plusieurs espèces à statut particulier telles que les espèces endémiques ivoiriennes (2 espèces) et Ouest africaines ( 35 espèces) et surtout celles de la liste rouge 
de l'UICN (27 espèces). Pour compléter l'étude de la végétation de la forêt, il faudrait réaliser des inventaires floristiques en prenant en compte la phénologie des espèces. C'est-à-dire réaliser des inventaires à différentes périodes de l'année. Sur la base d'un inventaire floristique plus conséquent, une analyse phytogéographique devrait être envisagée afin de préciser le statut phytogéographique du site étudié. Malheureusement, il existe 587 chefs d'exploitants qui exercent dans la forêt. Toutes ces infiltrations font que la forêt est en progressive dégradation. De nombreux produits forestiers non ligneux (PFNL) sont extraits de la forêt classée. II ressort des enquêtes que le commerce de ces produits est très florissant, même si les statistiques ne sont pas tenues. II est donc urgent d'impliquer

\section{RÉFÉRENCES BIBLIOGRAPHIQUES}

Adingra MMAO, 2013. Étude systématique et phytogéographique de la forêt classée de la Bamo (Côte d'Ivoire), Mémoire de DEA, UFR Biosciences Université Félix Houphouët-Boigny, Abidjan, Côte d'Ivoire, 47p.

Aké-Assi L, 1984. Flore de la Côte d'lvoire: étude descriptive et biogéographique, avec quelques notes ethnobotaniques. Thèse Doctorat, Université Nationale d'Abidjan, 1206 p.

Aké-Assi L, 1988. Espèces rares et en voie d'extinction de la flore de la Côte d'lvoire. Bot. Missouri Botanic Garden 25 : 461-463.

APG III, 2009. An update of the Angiosperm Phylogeny Group classification for the orders and families of flowering plants. Botanical Journal of the Linnean Society 161: 105-121.

Balaguru B, Britto SJ, Nagamurugan N, Natarajan D \& Soosatraj S, 2006. Identifyting Conservation priority zones for effective management of tropical forets in Eastern Ghats of India. Biodiversity and Conservation 15: 1529-1543.

Bakayoko A, 1999. Comparaison de la composition floristique et de la structure forestière de parcelles de la Forêt Classée de Bossématié dans l'Est de la Côte d'Ivoire. Mémoire de DEA, Université de Cocody -Abidjan (Côte d'Ivoire) 72 p.

Bertault JG, 1986. Étude de l'effet d'interventions sylvicoles sur la régénération naturelle au sein d'un périmètre expérimental d'Aménagement en forêt dense humide de Côte d'Ivoire. Thèse Doctorat, Université de Nancy (France), 254 p. davantage les populations riveraines dans la gestion des forêts en Côte d'Ivoire. En perspective, les sociétés INPROBOIS et la SODEFOR chargées de la gestion de cette forêt devraient rechercher à conserver la biodiversité et à protéger les sols et les eaux à l'intérieur de la forêt même si cela devrait passer par des déguerpissements. Ainsi, les objectifs environnementaux suivants pourraient recherchés : protection des massifs contre les défrichements; protection des sols (contre le ruissellement et l'érosion) ; protection des eaux (contre l'assèchement, la pollution, etc.) ; conservation des zones à importance pour la biodiversité ; protection de la faune ; reconstitution des zones dégradées et enfin des études et recherche.

Decocq G, 2003. Phytosociologie, syndynamique et archéologie du paysage. Contributii Botanice XXXVIII (2): 13-21.

Dupouey JL, Dambrine E, Laffite JD, Moares C, 2002. Irreversible impact of past land use on forest soils and biodiversity. Ecology 83: 2978-2984.

Guillaumet J-L, Adjanohoun E, 1971. La végétation de la Côte d'Ivoire. In : Avenard JM, Eldin E., Girard G, Sircoulon J, Touchebeuf P, Guillaumet JL, Adjanohoun $\mathrm{E}$ et Perraud A (eds). Le milieu naturel de la Côte d'Ivoire. Mém. ORSTOM 50 : 157-263.

Hoppe-Dominik B, 1999. L'état actuel des effectifs de grands mammifères dans l'ensemble du parc national de Taï. Direction de la protection de la nature, (Côte d'Ivoire), $66 \mathrm{p}$.

INPROBOIS, 2013. Plan d'aménagement de la forêt classée de la Besso 2003-2012. Rapport d'activité INPROBOIS, document non publié.

Kouamé N'F, 1998. Influence de l'exploitation forestière sur la végétation et la flore de la forêt classée du Haut-Sassandra (Centre-Ouest de la Côte d'Ivoire), Thèse Doctorat 3 Cycle, UFR Bioscience, Université de Cocody-Abidjan (Côte d'Ivoire), 227p.

Lebrun JP, Stork AL, 1991-1997. Énumération des plantes à Fleurs d'Afrique Tropicale. Conservatoire et Jardin Botaniques de la Ville de Genève, Genève (Suisse). Vol. 1 (249 pp.), vol. 2 (257 pp.), vol. 3 (341 pp.) et vol. 4 (711 pp.).

Mangenot G., 1955. Étude sur les forêts des plaines et plateaux de la Côte d'lvoire. Études Éburnéennes IV : 5-81. 
Myers N, Mittermeier RA, Mittermeier CG, da Fonseca GAB, Kent J, 2000. Biodiversity hotspots for conservation priorities. Nature 403: 853-858.

N'Da DH, Adou YCY, N'Guessan KE, Koné M, Sangne YC, 2008. Analyse de la diversité floristique du Parc National de la Marahoué, Centre-ouest de la Côte d'Ivoire. Afrique Science 4 (3) : 552-579.

Puig $\mathrm{H}$, 2001. La forêt tropicale humide. Belin, Paris (France), $448 \mathrm{p}$.

Raunkiaer C, 1934. The lifes forms of plants and statistical plant geography. Oxford University Press, London (Angleterre), $632 \mathrm{p}$.

Senterre B, 2005. Recherches méthodologiques pour la typologie de la végétation et la phytogéographie des forêts denses d'Afrique tropicale. Thèse de Doctorat, Université Libre de Bruxelles (Belgique), $345 \mathrm{p}$.

UICN (International Union for the Conservation of the Nature), 2009. IUCN Red List of Threatened Species. Version 2009.2. [Online] Available: www.iucnredlist.org

White $F, 1986$. La végétation de l'Afrique. Recherche sur les ressources naturelles. ORSTOM UNESCO, Paris (France), XX: 384. 